\title{
Computer-Based Wireless Advertising Communication System (CBWACS)
}

\author{
doi:10.3991/ijim.v3s2.827
}

\author{
Yahya S. H. Khraisat, Anwar Al-Mofleh \\ Al-Balqa Applied University/ Al-HusonUniversity College, Amman, Jordan
}

\begin{abstract}
In this paper we developed a computer based wireless advertising communication system (CBWACS) that enables the users to advertise the information they want to display from their own offices to the screen in front of the customer via wireless communication system. This system consists of two PIC microcontrollers, transmitter, receiver, LCD, serial cable and antenna. The main advantages of the system are the wireless structure and the digital communication techniques. So this system is less susceptible to noise and other interferences.
\end{abstract}

Index Terms-Computer; Microcontroller; Amplitude Shift Keying; Transmitter; Receiver; antenna.

\section{INTRODUCTION}

The idea of CBWACS came from the need to visualize some certain information in public by typing it in the user's display and sending it via wireless system to main display. The system provides a kind of dialog and interaction between the company and its customers.

Banks for example, may want to improve their customer services by putting a large, common and well seen monitor to display and inform their clients of when the bank closes, opens, what services are available and the newest released offers.......etc.

From what had been described we can see that CBWACS shows us a clear view of what the future would look like; by its capability of establishing an interaction between companies and their clients, that save time and money.

Systems based on analogue communication techniques are more sensitive to the interferences. So we designed our system to have more handling capability to noise by modulating the base-band signals to band-pass signals and transmit the data using digital techniques. The advantages of digital communications are:

1. Digital signals are easily represented (ones or zeros) compared with analogue ones.

2. Digital circuits are less subject to distortion and interference unlike analogue circuits, because binary digits operate in one of two cases i.e. fully on or fully off.

3. More reliable and can be produced at lower cost than analog circuits.

\section{A. Structure of $C B W A C S$}

The structure of a CBWACS system consists of the following essential components: two PIC microcontrollers, transmitter, receiver, LCD, serial cable, and antenna.

We will discuss each component in the next sessions.
CBWACS structure consists of two parts:

1. transmitting part $(\mathrm{PC}, \mathrm{MAX} 232 \mathrm{C}, \mathrm{PIC}$, transmitter, antenna)

2. receiving part (antenna, receiver, PIC, LCD)

Figure 1 represents the block diagram of our wireless advertising system
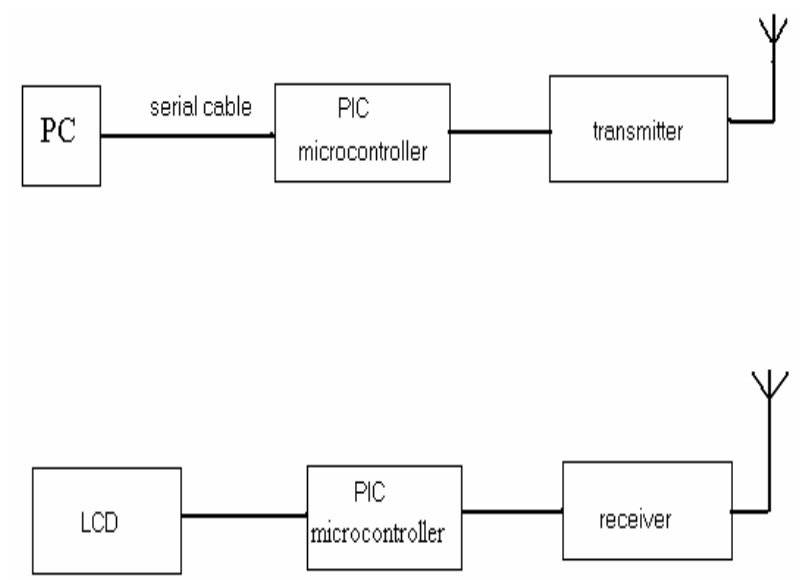

Figure 1. Block diagram of Wireless Advertising System

\section{B. Data Processing Overview}

The system has two parts transmitting and receiving. We shall view the data processing for each element in the system [1]:

The transmitting part of CBWACS does the following:

1. Receive the textual message from the serial cable in ASCII code (0-12 volt).

2. MAX232C interfaces the incoming signals to PIC (05 volt).

3. The PIC microcontroller converts the ASCII bits into pulse width modulating (PWM).

4. The output of the PIC is fed to the transmitter.

5. The transmitter sends the modulated data to the receiving part of the system through the antenna.

The receiving part of CBWACS does the following:

1. When the RF signal is picked up from space by a receiving antenna, a voltage is induced into the antenna (a conductor). The RF voltages induced into the receiving antenna are then passed into the receiver and converted back into the transmitted RF information.

2. The receiver received band-pass signals that were transmitted and recovered the base-band signal. This signal is then fed to the PIC microcontroller. 
3. The PIC microcontroller converts the incoming PWM back to their original forms.

4. The PIC microcontroller then sends the message signal in ASCII code to the LCD.

5. The LCD displays the received data.

In other words the first PIC microcontroller programmed to convert its input serial data from ASCII into PWM, and send it to the transmitter. The second PIC microcontroller is programmed to convert the received waveform back into its basic pulse representation (PWM) then its code contains some instructions to control the LCD.

The PIC in the transmitting part converts the incoming serial data in ASCII to a PWM. Every letter in the textual message has its own separate identifying width so as to minimize the bit error probability in the detection process at the receiving part of the system. Then those PWM sequences are sent to the receiving part of the system through the transmitter after modulating it to a band-pass signal in the form of ASK -amplitude shift keying-.

The receiving part of our system which has other PIC microcontroller receives whatever the transmitter sends. The demodulation/detection process takes place to reconstruct the original message signal. Then the PIC converts the incoming serial bits into its original form (extracting the letters). The letters representing the user textual message are fed to the LCD screen.

\section{Transmitting Part Data Processing}

The transmitting part of the system consists of the computer, MAX232C, PIC, and the transmitter. The transmitter processes the data through its terminals from input to output in four separated stages: computerMAX232C, MAX232C-PIC, PIC-transmitter and transmitter-antenna).

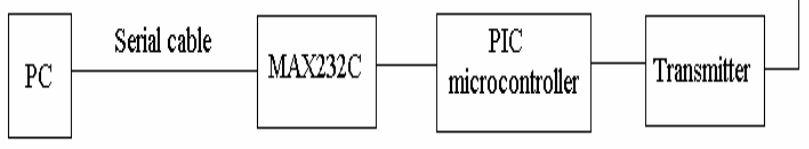

Figure 2. Transmitting part components

The primary subject in this session is the processing of the message signal. The block diagram which represents the stages at which the data would be flown within the transmission part of the CBWACS system is shown in figure 3 .

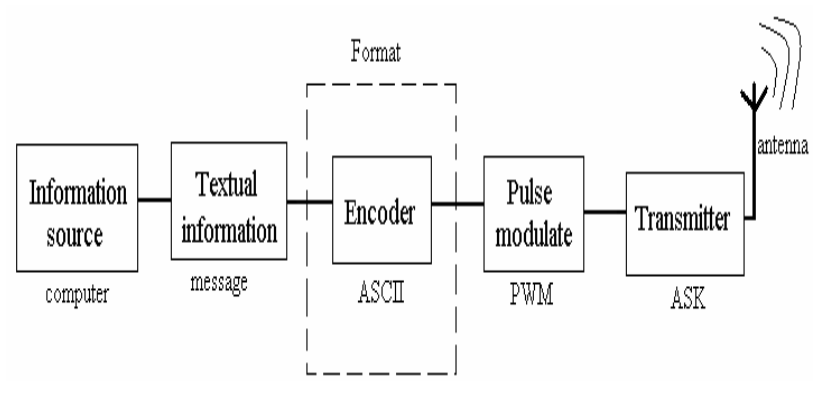

Figure 3. Overall data processing in the transmitting part

\section{A. Formatting Textual Data (Character Coding)}

The goal of the first essential signal processing step is to insure that the message (or source signal) is compatible with digital processing. Textual information is transformed into binary digits by the computers' CPU.

The computers' keyboard is the information source of the system that provides a textual data entrance to the computer system. It could be encoded by the ASCII code into binary sequence [2].

The computer sees the user's message as a sequence of binary ones and zeros formed of every letter ASCII code representation. The USART terminal of the MIKROBASIC attaches that sequence to the computer transmitting unit that is the computers serial port. This port sends the message binary sequence through the serial cable with amplitudes of $0-12$ volts.

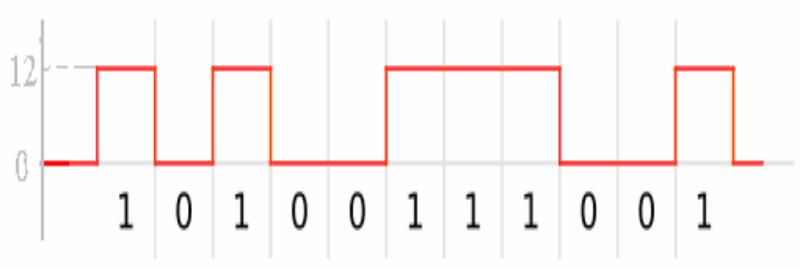

Figure 4. USART terminal

Then the sequence goes to the MAX232C, which is the interface of the PIC microcontroller. MAX232C converts the incoming sequence amplitudes from (0-12 volts) to (05 volts) so as PIC could handle and process it.

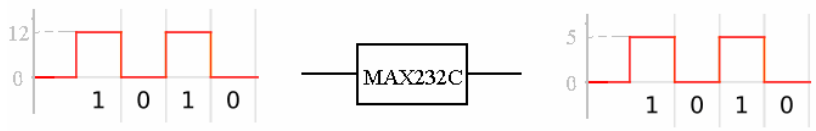

Figure 5. Pulse modulation

Conversion from a bit stream to a sequence of pulse waveforms takes place in the block labelled as pulse modulation. As the message flows in the system, arriving to the first PIC; the sequence is read in the following manner: the PIC microcontroller reads the sequence letters letter by letter. This message stored in the PIC microcontroller, RAM in parallel. Then the PIC microcontroller reads and processes the sequence by its algorithm. The algorithm was designed to give the letters that represent the message a unique width. In other words the PIC microcontroller's algorithm is designed to do a kind of modulation known as PWM, involving varying each letter duty cycle to discriminate it from other letters in the message.

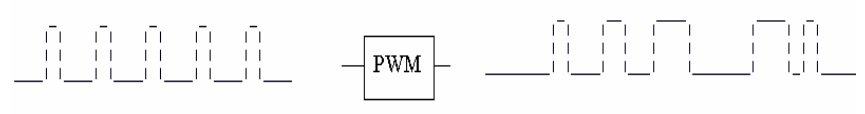

Figure 6. Pulse-Width Modulation [3]

The width that every letter occupies is affected by the baud rate that the transmitter used. After the PIC microcontroller had modulated the message pulses, it would be sent to the transmitter serially. The PIC microcontroller final duty is to perform a parallel to series conversion. 


\section{B. Data Transmission}

The transmitter is an electronic device which with the aid of an antenna propagates an electromagnetic signal. The transmitter converts incoming base-band signal to a band-pass signal which will be transmitted to the receiver by a kind of band-pass modulation technique known as ASK -amplitude shift keying. ASK is a form of band-pass modulation which represents digital data as variations in the amplitude of a carrier wave [3].

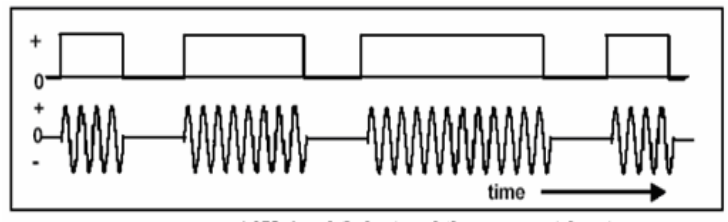

an ASK signal (below) and the message (above)

Figure 7. Amplitude Shift Keying

The amplitude of an analog carrier signal varies in accordance with the bit stream (modulating signal), keeping frequency and phase constant. The level of amplitude can be used to represent binary logic $0 \mathrm{~s}$ and $1 \mathrm{~s}$. We can think of a carrier signal as an ON or OFF switch. In the modulated signal, logic 0 is represented by the absence of a carrier, thus giving OFF/ON keying operation and hence the name (OOK) was given. The transmitter gives a maximum baud rate of $40 \mathrm{kbps}$, but as we sent a textual data we satisfied with a less baud rate than what the transmitter gives. If we use transmitter at 1 kbps, the letter "a" will need (0-1) ms to be transmitted. Adding some more width to the next letter say $0.5 \mathrm{~ms}$, the letter " $z$ " have the longest time that is approximately around 0.03 second. Assuming that the message has a hundred letters all of them are " $z$ ", then the message needs three seconds to appear on the receiving part display unit. This is a very long time that the users are unhappy to see. So we used the transmitter baud rate at a higher range. If we make the transmitter baud rate $20 \mathrm{kbps}$, the letter "a" needs a 0,5 microseconds, and thus the letter " $\mathrm{z}$ " needs 0.13 microseconds plus some interval gab to reduce the errors, say 0.14 microseconds. So if the message contains a hundred letters all of are " $z$ ", it needs 14 microsecond to be transmitted, which is better from the fist one.

Finally, the transmitter sends its band-pass modulating signal to the antenna. The antenna is used either for radiating electromagnetic energy into space or for collecting it from space. The use of an antenna improves the range of the RF link and reduces the susceptibility to noise. The choice of antenna and its positioning can affect the performance of the RF link. The best place to mount the antenna is outside the box away from all sources of interference, metal items and ground planes.

\section{RECEIVING PART DATA Processing} parts:

The receiving part of the system has the following

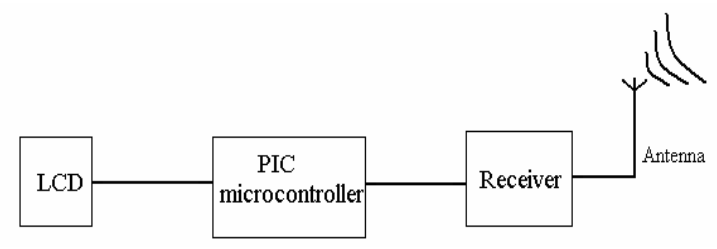

Figure 8. Receiving part components
We are interested in recovering the original message signal. Since band-pass model of the detection process is virtually identical to the base-band model, the final detection step takes place, according to the equivalence theorem which states:

Performing band-pass linear signal processing followed by heterodyning the signal to base-band, yield the same result as heterodyning the band-pass signal to base-band, followed by base-band linear signal processing [4].

The term "heterodyning" refers to a frequency conversion or mixing process that yields a spectral shift in the signal.

The received signal will be processed in the receiving part of the system as shown in the following block diagram.

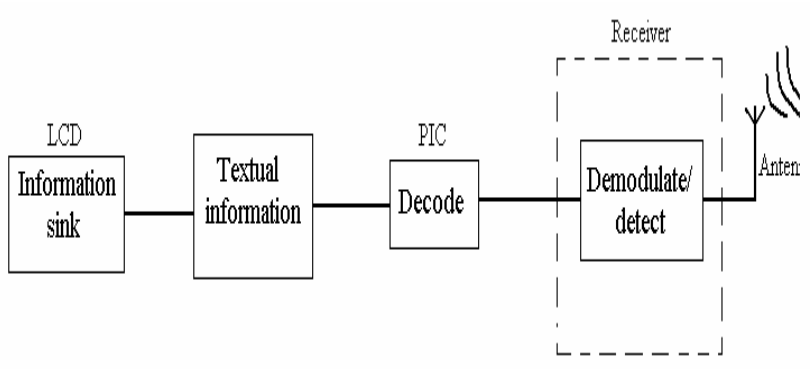

Figure 9. The over all data processing in the receiving part

When the RF signal is picked up from space by a receiving antenna, a voltage is induced into the antenna. The RF voltages induced into the receiving antenna are then passed into the receiver and converted back into the transmitted RF information. In other words on the receiving part, electromagnetic energy is converted into electrical energy by the antenna and fed into the receiver.

\section{A. Demodulation and Detection}

The channel typically causes the received pulse sequence to suffer from inter symbol interference (ISI) and thus appears as smeared signal, not quite ready for sampling and detection. That is the receiver signal is distorted, and the receiver will deal with that smeared signal. The receiver has to fix and sample it in a right way so as to recover the original message signal.

The goal of the demodulator (receiving filter) is to recover a base-band pulse with the best possible of signalto-noise ratio (SNR), free of any ISI. Equalization is a technique used to help accomplishing this goal. The equalization process is not required for every type of communication channel.

However, since equalization embodies a sophisticated set of signal-processing techniques, making it possible to compensate for channel induced interface, it is an important area for many systems.

During a given signalling interval $\mathrm{T}$, a binary base-band system will transmit one of two waveforms, denoted g1 $(\mathrm{t})$ and g2 (t). Similarly, a binary band-pass system will transmit one of two waveforms, denoted s1 (t) and s2 (t).

Since the general treatment of demodulating and detection are essentially the same for base-band and band-pass systems, we used si ( $\mathrm{t}$ ) here as a generic designation for a transmitted wave form.

Then any binary transmitted signal over a symbol interval $(0, T)$ is represented by 


$\begin{array}{ccc} & \mathrm{Si}(\mathrm{t})= \\ \mathrm{s} 1(\mathrm{t}) & , 0<\mathrm{t}<\mathrm{T} & \text { for binary } 1 \\ \mathrm{~s} 2(\mathrm{t}) & , 0<\mathrm{t}<\mathrm{T} & \text { for binary } 0\end{array}$

The received signal $r(t)$ is degraded by the noise $n(t)$ and by the impulse response of the channel hc $(\mathrm{t}) . \mathrm{r}(\mathrm{t})$ is written as

$$
r(t)=s i(t) * h c(t)+n(t)
$$

Where $\mathrm{n}(\mathrm{t})$ is here assumed to be a zero mean additive white Gaussian noise AWGN process, and * represents the convolution operation.

Figure 10 shows the typical demodulation/detection function of a digital receiver.

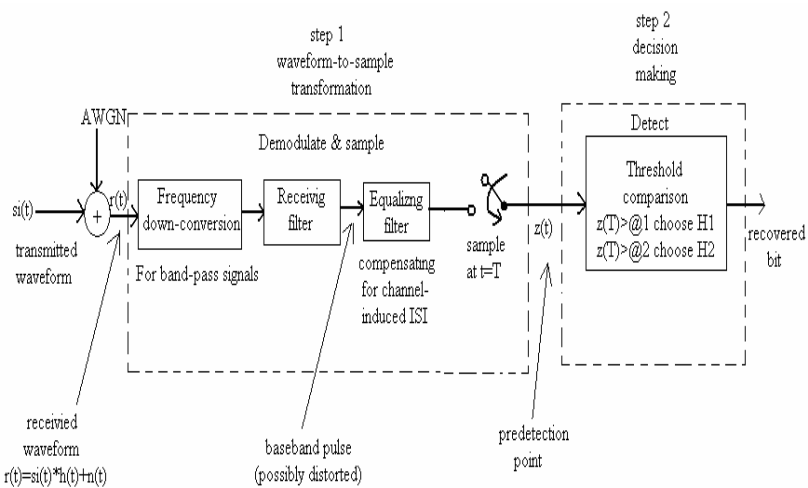

Figure 10. The two basic steps in the demodulation/detection of digital signals

We define demodulation as recovery of a waveform (to an undistorted base-band pulse). We designated detection as the mean of decision-making process of selecting the digital meaning of the waveform.

The frequency down-conversion block, shown in the demodulator portion of the figure 10 , performs frequency translation for band-pass signals operating at some radio frequency $(\mathrm{RF})$.

The receiving filter (essentially the demodulator) performs waveform recovery in preparation for the next important step-detection. The filtering and the channel at the transmitter typically cause the received pulse sequence to suffer from ISI, and thus it is not quite ready for sampling and detection.

The goal of the demodulator (receiving filter) is to recover a base-band pulse with the best possible signal-tonoise ratio (SNR), free of any ISI. The optimum receiving filter for accomplishing this is called a matched filter or correlator [5]. An optional equalizing filter follows the receiving filter. This filter is only needed for those systems where channel induced ISI can distort the signals.

The received filter and the equalizer filter are shown separately to indicate the importance of their functions in demodulating the signals. In most cases, however, when an equalizer is used, a single filter would be designed to incorporate both functions and thereby compensate for the distortion caused by both the transmitter and the channel. Such composite filter is sometimes referred to simply as the equalizing filter or the receiving and equalizing filter.

In the demodulation/detection process there are two steps namely step 1 and step 2 . In step 1 , the waveform-to- sample transformation, is made up of the demodulator followed by a sampler. At the end of each symbol duration $T$, the output of the sampler yield a sample $Z(T)$ called the test statistics. Z (T) has a voltage value proportional to the energy of the received symbol and that of the noise [4].

In step 2 a decision (detection) is made regarding the digital meaning of that sample. We assume that the input noise is a Gaussian random process and that the receiving filter in the demodulator is linear. A linear operation performed on a Gaussian random process produces a second Gaussian random process thus; the filter output noise is Gaussian. The output of step 1 yields the test statistics

$$
\mathrm{Z}(\mathrm{T})=\mathrm{ai}(\mathrm{T})+\mathrm{n} 0(\mathrm{~T}) \quad \mathrm{i}=1,2
$$

Where ai $(\mathrm{T})$ is the desired signal component, and $\mathrm{n} 0$ $(\mathrm{T})$ is the noise component. The noise component is a zero mean Gaussian random variable, and thus $\mathrm{z}(\mathrm{T})$ is a Gaussian random variable with a mean of either alor a2 depending on whether a binary one or zero was sent. The probability density function (PDF) of the Gaussian random noise $\mathrm{n} 0$ is $\mathrm{p}(\mathrm{z} / \mathrm{n} 0)$, like the PDFs of s1and $\mathrm{s} 2$.

The last two conditional pdfs are illustrated in the next figure. The right most conditional PDF, $\mathrm{p}(\mathrm{z} / \mathrm{s} 1)$ called the likelihood of s1 which illustrates the probability density function of the random variable $\mathrm{z}(\mathrm{T})$, given that symbol s1 was transmitted. Similarly, the left most conditional PDF, $\mathrm{p}(\mathrm{z} / \mathrm{s} 2)$ called the likelihood of $\mathrm{s} 2$, illustrate the probability density function of the random variable $\mathrm{z}(\mathrm{T})$, given that symbol s2 was transmitted.

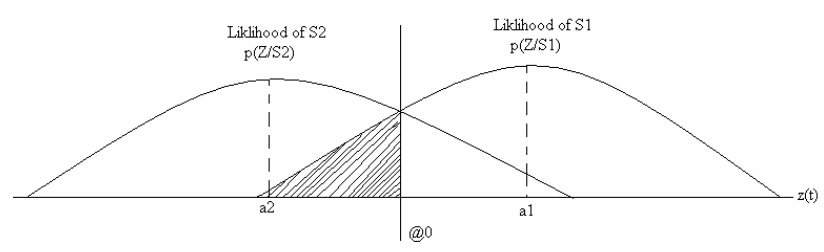

Figure 11. Conditional probability density functions: $\mathrm{p}(\mathrm{Z} / \mathrm{S} 1)$ and $\mathrm{p}$ $(\mathrm{Z} / \mathrm{S} 2)$

After the received wave form has been transformed to a sample, the actual shape of the wave form is no longer important. All waveform types that are transformed to the same value of $\mathrm{z}(\mathrm{T})$ are identical for detection purpose. Later it will be shown that the matched filter (receiving filter) maps all signals of equal energy into the same point $z(T)$, therefore the receiving signal energy not its shape is the important parameter in the detection process. This is why the detection analysis for base-band signals is the same as that for band-pass signals. Since z (T) a voltage signal that is proportional to the energy of the received symbols, the larger the magnitude of $\mathrm{z}(\mathrm{T})$, the more error frees the decision-making process.

In step 2, detection is performed by choosing the hypothesis that result from the threshold measurement

$$
\begin{aligned}
& \mathrm{Z}(\mathrm{T})>\text { choose H1 } \\
& \mathrm{Z}(\mathrm{T})<@ \quad \text { choose H2 }
\end{aligned}
$$

When $\mathrm{H} 1$ and $\mathrm{H} 2$ are the two possible (binary) hypotheses, the inequality relationship indicates that hypothesis $\mathrm{H} 1$ is chosen if $\mathrm{z}(\mathrm{T})>@$, and hypothesis $\mathrm{H} 2$ is chosen if $\mathrm{z}(\mathrm{T})<@$, if $\mathrm{z}(\mathrm{T})=@$, the decision can be an arbitrary one. Choosing $\mathrm{H} 1$ is equivalent to deciding that 
s1 (t) was sent and hence a binary one is detected. Similarly, Choosing $\mathrm{H} 2$ is equivalent to deciding that $\mathrm{s} 2(\mathrm{t})$ was sent and hence a binary zero is detected.

\section{1) The Matched Filter}

A matched filter [5] is a linear filter designed to provide the maximum signal-to-noise power ratio at its output for a given transmitted symbol waveform. Consider that a known signal $\mathrm{s}(\mathrm{t})$ plus AWGN $\mathrm{n}(\mathrm{t})$ are the inputs to a linear, time invariant (receiver) filter followed by a sampler, as shown in figure 10 . At time $\mathrm{t}=\mathrm{T}$, the sampler output $\mathrm{z}(\mathrm{T})$ consists of a signal component ai and a noise component n0. Manipulation for the impulse response of the matched filter yields the following

$$
\mathrm{H}(\mathrm{t})=\left\{\begin{array}{lr}
K s(T-t) & 0 \leq t \leq T \\
0 & \text { elsewhere }
\end{array}\right.
$$

Thus, the impulse response of a filter that produces the maximum output signal-to-noise ratio is the mirror image of the message signal $s(t)$, delayed by the symbol time duration T. Without the delay the response $s(-t)$ is unrealizable because it describes a response as a function of a negative time. Figure 12 illustrates the matched filter's basic property: the impulse response of the filter is a delayed version of the mirror image (rotated on the $t=0$ axis) of the signal waveform.

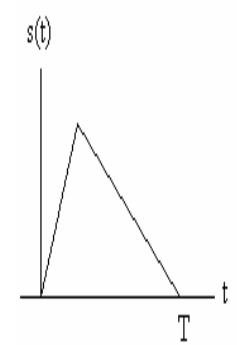

sigha watiofin

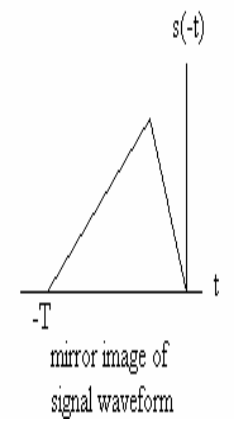

Figure 12. Matched filter characteristics

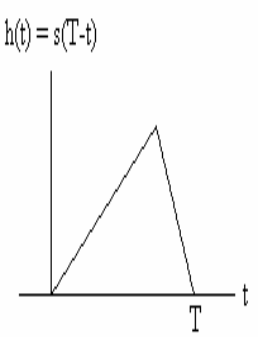

impulse respontice of matche filter

\section{B. Decoding the Recovered (Base-Band) Signal}

The original base-band signal (Textual information) is sent to the PIC microcontroller bin serially and stored in the PIC microcontroller RAM. Then PIC microcontroller (No.2) deals with the arriving signal with its algorithm in the following manner:

The algorithm was written in order to decode the signal from its binary form to its basic meaning. The sequence that is letters, and sends them to the display unit of our system, that is the LCD. The PIC microcontroller converts the serial message signal into parallel so as to deal with each letter separately.

As the ASCII code gives a unique pulse representation for each letter, we had modulated those pulses in the PIC microcontroller with the PWM. ASCII code gives each letter a unique width to discriminate it from the other letters in the message in order to reduce the probability of errors and ISI. Now the PIC microcontroller algorithm recovers each letter with its determined width in the transmitting part plus extra small width. To insure that we recovered the exact transmitted letter without any errors at all. We could consider that process as a form of error immunity for our system.

\section{Passing Textual Information-to-Information Sink}

After the PIC microcontroller's algorithm had finished its duty, the original message combining all the letters would be recovered. The algorithm sends that message to its output bin serially to the LCD. The mikrobasic is software that we used to program the PICs.

\section{CBWACS COMPONENTS CONNECTION}

In this paragraph we represent the necessary hardware which we used. We will talk about the necessary connection to support the system software. In part one, we showed that the system contains two parts, transmission part and receiving part. Now we will demonstrate the components of each part.

\section{A. Transmission Part}

The transmission part has the following components

1. Power supply

2. Oscillator

3. Rest

4. RS 232 interface

5. PIC 16F877A

6. Transmitter

\section{1) Power supply}

The power supply is needed in the two parts (transmission and receiving). The input power supply is applied to systems that have voltage range of $\mathrm{V}$ equal 15 Vor less than $13 \mathrm{~V}$ and its maximum current is $20 \mathrm{~mA}$. We can choose any value in this range to get $5 \mathrm{~V}$ from the regulator, as shown in figure 12 [6].

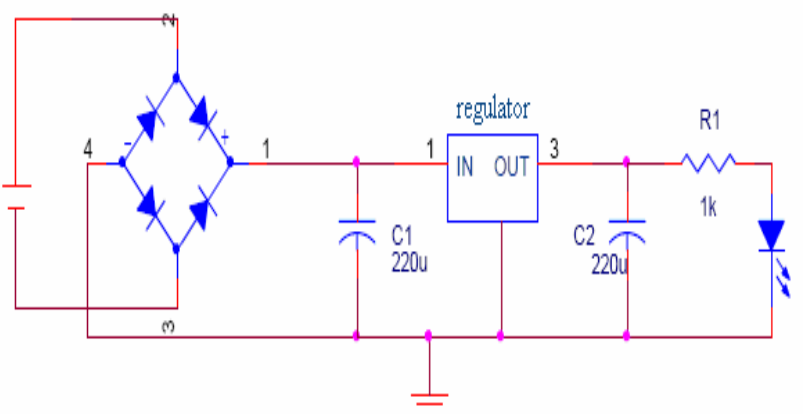

Figure 13. Power Supply Circuit

Generally microcontrollers are based on CMOS technology.

The operating voltage is connected to $\mathrm{Vdd}$ (pin \#11).

In the transmitter the operating voltage $5 \mathrm{~V}$ is connected to pin $\# 4$, in $\max 232$ the $5 \mathrm{~V}$ is connected to pin $\# 2$, and pin \#16. Pin \#2 is connected to a capacitor to keep the voltage stable.

\section{2) Oscillator}

We used crystal oscillator for the PIC16F877A at 4 MHZ. The oscillator used as an external clock. Increasing the clock speed is proportional to increasing the size of the incoming data. The steps needed to complete an instruction are 4 clocks. The 4 clocks are equal to one cycle. So to get the instruction speed, the four clocks are divided by $4 \mathrm{MHZ}$ equals lus (1us is the duration to complete the instruction) [7]. 


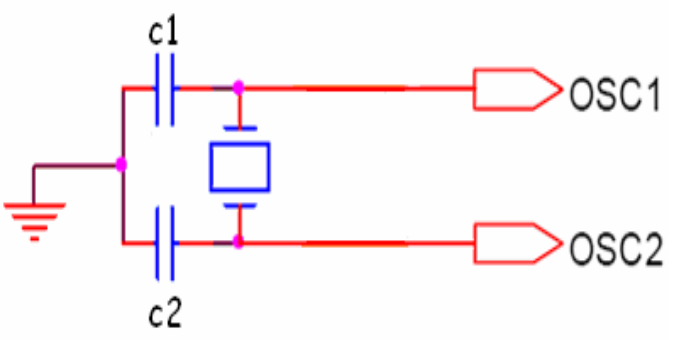

Figure 14. Oscillator circuit

The smoothing capacitors $\mathrm{C} 1$ and $\mathrm{C} 2$ are used to reject any attenuation.

3) Reset

Reset is used to restart the downloaded program in PIC, in other words it is used to initiate the external clock. A click switch is connected to the _MCLR pin $\left(1^{\text {st }}\right.$ pin in the PIC). After polling it up with a $10 \mathrm{~K} \Omega$ resistance and smoothed by $0.1 \mathrm{uF}$ capacitor. R2 is used to reduce the back current form capacitor. It is required that you consider the Reset button in our design.

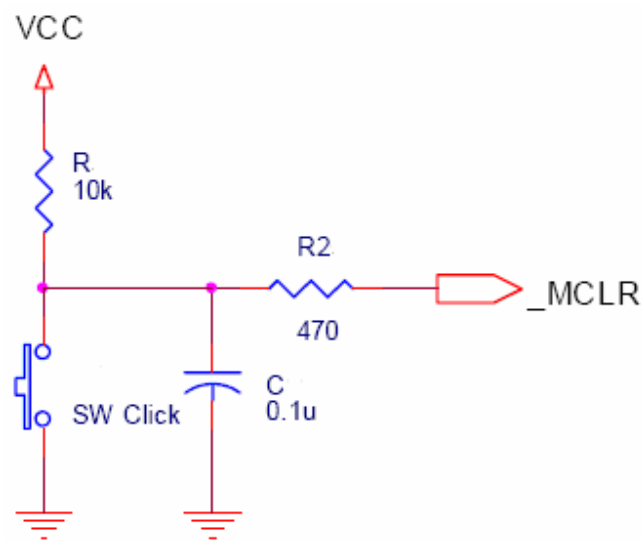

Figure 15. Reset circuit

\section{4) RS 232 Interface}

The binary pulses which came from the serial port had the amplitudes 0 and +12 volts. The PIC microcontrollers don't deal with 0 and +12 volts, they are dealing with 0 and +5 volt. So we used $\max 232 \mathrm{c}$ to convert the incoming pluses to 0 and +5 volts. So RS 232 interface, fig15, supports the USART module.

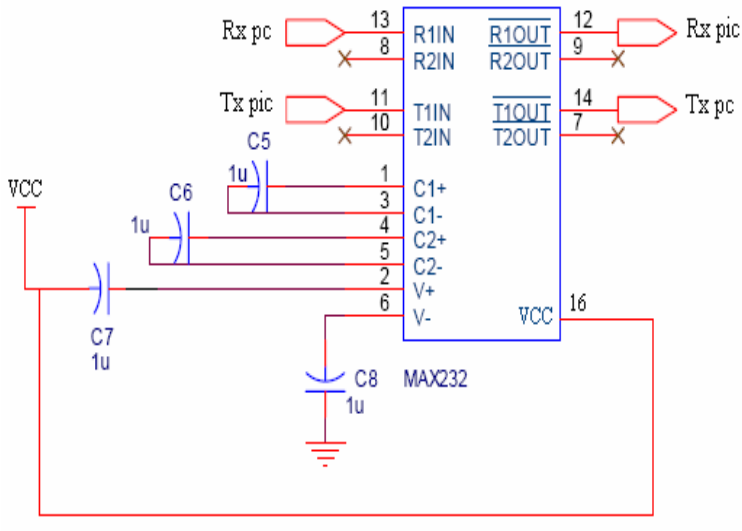

Figure 16. The (RS232 interface) circuit

\section{5) $\mathrm{PIC} 16 \mathrm{~F} 877 \mathrm{~A}$}

PIC is a family of RISC microcontrollers made by Microchip Technology. PIC stands for "Peripheral Interface Controller" Or "Programmable Intelligent Computer". We are interesting in the 16F877A type of the wide range of the PIC microcontrollers, which is amid range family of a various families of PIC microcontroller that consist of 40 pins. The $\mathrm{F}$ in the name $16 \mathrm{~F} 877 \mathrm{~A}$ generally indicates that the PIC microcontroller uses flash memory and can be erased electronically. The number 16 in front of a name shows the family range that this PIC belongs to (low, medium, high). The last three numbers refer to a certain features the PIC have. The last letter A shows the category of improvement that was done to the PIC.

\section{6) Transmitter}

In this work we used a $434 \mathrm{MHZ}$ OOK (on-off keying) AM transmitter with external antenna. Fig 16 illustrates the connection between transmitter and PIC.

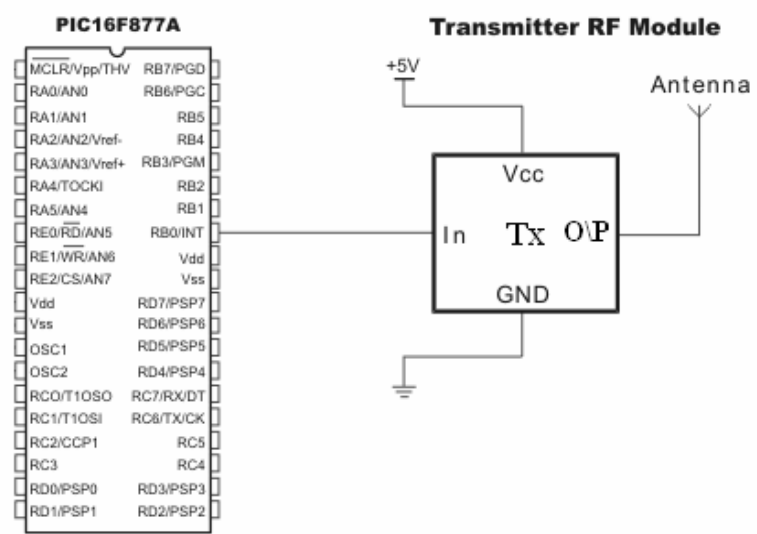

Figure 17. Transmitter connection

\section{7) Transmitting Part Overall Connection}

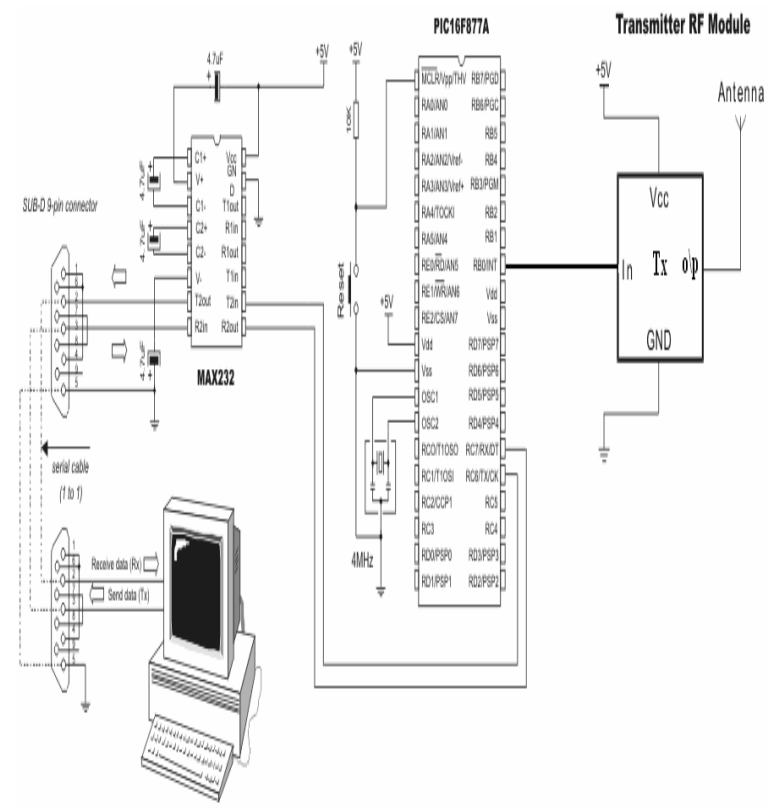

Figure 18. Transmitting part 


\section{B. Receiving Part}

This part has the following components:

1. Power supply

2. Oscillator

3. Reset

4. PIC 16F877A

5. Receiver

6. $\operatorname{LCD}(16 \times 2)$

The power supply circuit, oscillator circuit, and Reset circuit are the same as what we have discussed in the transmission part.

1) Receiver

Fig 19 illustrates the hardware connection of this receiver.

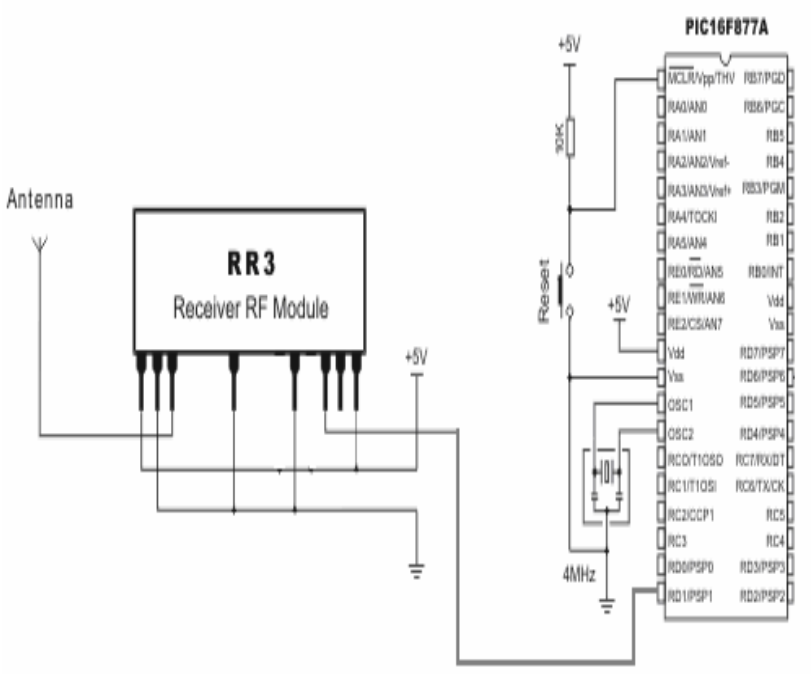

Figure 19. Receiver connection

\section{2) LCD (Liquid Crystal Display)}

A liquid crystal display (LCD) is a thin, flat display device made up of any number of arrayed pixels. It is prized by engineers because it uses very small amounts of electric power. LCD contains a small processor and small memory that is controlled by the LCD. There are two kinds of LCD. The first uses a serial data bus and the other uses a parallel data bus. In our work we used parallel data bus because it's simple and fast read $\mid$ write. We will use the LCD to view the output. The LCD that we used is similar to the one in the previous picture. It has two rows and 16 characters, as shown below.

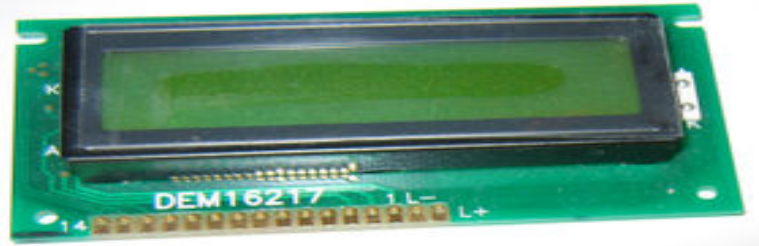

Figure 20. A general purpose alphanumeric LCD, with two lines of 16 characters

The hardware connection of LCD with PIC is shown in figure 21 .

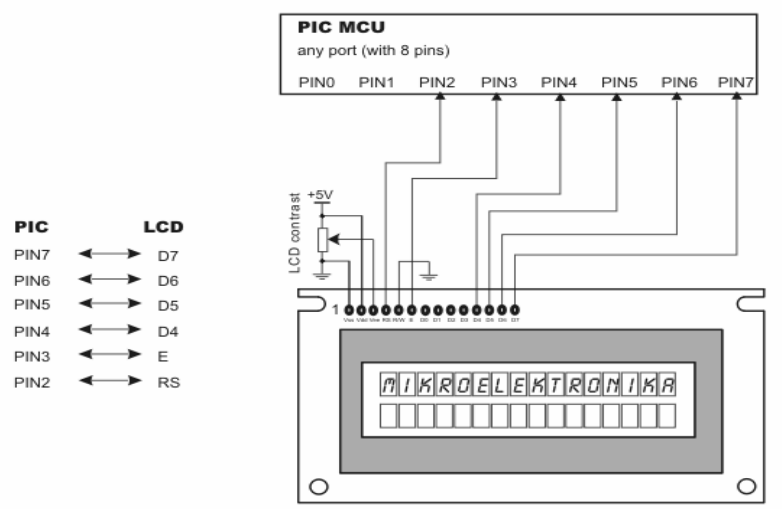

Figure 21. LCD connection

We can write and read from LCD by using two modes; eight bit mode and four bit mode. The general mode to be displayed is eight bits long and is sent to the LCD either four or eight bits at a time. If four bits mode is used, two nibbles of data are sent to make up a full eight bits transfer (sent high four bits and then low four bits with an E clock pulse with each nibbles). This mode has the disadvantage of making errors. So we used the four bit mode that is a reliable method to accomplish our goal. For this work there is no reason to read from LCD, therefore the R/W usually tied to ground. We used the potentiometer wired as a voltage divider to specify the contrast of the character on the LCD screen.

\section{3) Receiving Part Overall Connection}

The general circuit for the receiving part is shown in figure 23 .

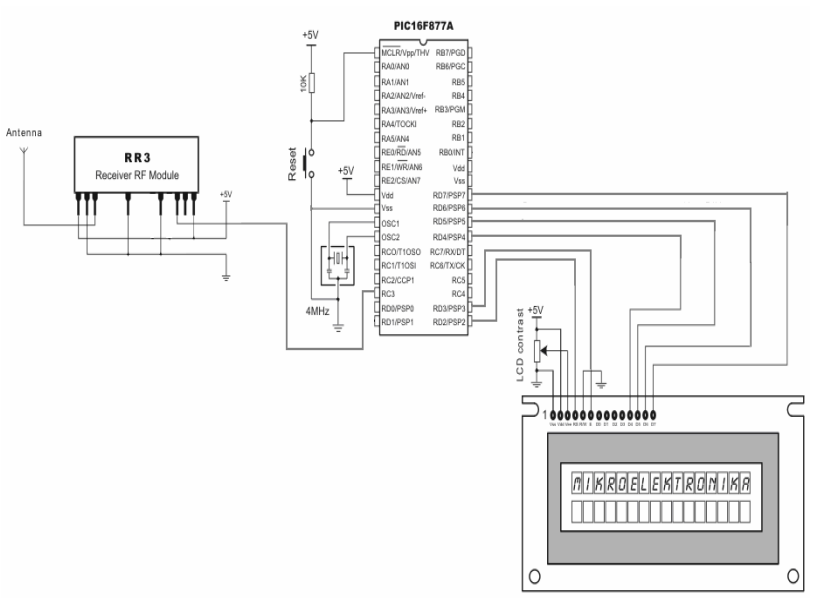

Figure 22. Receiving Part

\section{REFERENCES}

[1] Yahya S. H. Khraisat, Anas Al-Kurdi, Nimer Al-Shalabi and Salah-Eddin Mahasneh, " Local Area Mobile - System Communication (LAMS)", 3rd International conference on Interactive Mobile and Computer Aided Learning IMCL 2008, Princess Sumaya University for Technology, 16-18 April 2008, Amman, Jordan.

[2] Thomas L. Floyd, Digital Fundamentals, Prentice Hall, Eight edition, 2003.

[3] Haykin, S., Communication Systems, John Wiley \& sons, Inc., New York, 2000.

[4] Sklar, B., Digital Communications Fundamentals and Applications, Prentice Hall P T R, New Jersey, 2001.

[5] Merrill I. Skolnik, Introduction to Radar System, 3rd edition. New York: McGraw-Hill, 2001 


\section{COMPUTER-BASED Wireless AdVERTISING COMMUNICATION SYSTEM (CBWACS)}

[6] Thomas L. Floyd, Electronic Devices, Prentice Hall, Six edition, 2003.

[7] Neaman, Microelectronics Circuit Analysis and Design, Mc Graw Hill Inc, 2007.

[8] Millman, J., Microelectronics, Mc Graw Hill Inc, 1987.

[9] AUR.EL, Wireless System, Italy, available: www.aurel.it.

\section{AUTHORS}

Yahya S. H. Khraisat is an associated professor of the Electrical and Electronics Department, Al-Balqa Applied
University/ Al-Huson University College, Jordan, P.O. Box 1375, Irbed 21110. (E-mail: yahya@huson.edu.jo).

Anwar Al-Mofleh is an engineer of the Electrical and Electronics Department, Al-Balqa Applied University/ AlHuson University College, Jordan, P.O. Box 1375, Irbed 21110. (E-mail: Anwar1971@yahoo.com).

Submitted February 1st 2009. Published as resubmitted by the authors on 28 October 2009. 